\title{
The Polarization Potential as a Probe into Interstellar Matter
}

\author{
Amaury de Kertanguy \\ LERMA, Observatoire de Paris-Meudon Place Jules Janssen, Meudon, France \\ Email: amaury.dekertanguy@gmail.com
}

How to cite this paper: de Kertanguy, A. (2021) The Polarization Potential as a Probe into Interstellar Matter. Journal of Applied Mathematics and Physics, 9, 2623-2640.

https://doi.org/10.4236/jamp.2021.911169

Received: September 15, 2021

Accepted: November 2, 2021

Published: November 5, 2021

Copyright (c) 2021 by author(s) and Scientific Research Publishing Inc. This work is licensed under the Creative Commons Attribution International License (CC BY 4.0).

http://creativecommons.org/licenses/by/4.0/

\begin{abstract}
By simple arguments, it is shown how alkaline types of atoms can be seen in dense molecular clouds depending on the average temperature of such regions of the Universe. This paper predicts infrared lines of such atoms that could be seen in molecular clouds. The theory is developed in the first part and the predicted experimental results will follow. It results that the alkaline atoms modelized using the short-range polarization potential are found in quantum states $n_{*}>2$.
\end{abstract}

\section{Keywords}

Interstellar Matter, Alkaline Atoms, Giant Molecular Clouds

\section{Introduction}

It is established for a long time (1926) that one can model atomic structures of alkaline species adding to the Coulomb potential an attractive short-range potential: $V_{p}(r)=-\frac{e^{2}}{2} \frac{\alpha_{D}}{r^{4}}$ (It acts the same sign as the Coulomb potential) [1].

Our main purpose is to show that these corrections coming from the modification of the core structure in their neutral states lead to observable radiation energy in the infrared domain.

When one considers the polarization potential, it could be detectable in low-temperature parts of the universe that contain atoms, molecules and dust, it is known that these temperatures vary between $50 \mathrm{~K} \geq T_{\text {clouds }} \leq 500 \mathrm{~K}$ for molecular clouds of known or estimated densities (giant interstellar molecular clouds).

The infrared survey of molecular clouds exits since 2004 with the ISO instrument or later with the Herschel satellite (2009) [2], molecular lines are found in 
Orion cloud and are identified: $\mathrm{H}_{2} \mathrm{O}, \mathrm{CO}_{2}, \mathrm{CH}_{4}, \mathrm{NH}_{3}, \mathrm{CO}$ and neutral silicium (S). The prospect of this work is to foresee infrared lines (possibly detectable) emitted by alkaline atoms that exist in molecular clouds, with accepted abundances of these regions of the Universe.

The emission of infrared light, coming from these atomic transitions is obtained with effective quantum numbers: $n_{*}=n-\delta$ with $2 \leq n_{*} \leq 9$.

For the theoretical part of this work and subsequent calculations the hydrogen ionization energy $I_{H}=13.616 \mathrm{eV}$ is used to measure the effect of the atom core on energy levels $-\frac{e^{2}}{2} \frac{\alpha_{D}}{r^{4}}$.

$\alpha_{D}$ are the different static dipolar polarizabilities of the atoms, estimates of these quantities exist for elements: $\mathrm{Li}, \mathrm{Mg}, \mathrm{Na}, \mathrm{Cs}, \mathrm{K}, \mathrm{Ca}$ [3] [4].

\section{Dealing with Realistic Molecular Clouds Environment}

We consider the classical value of the $\left\langle\frac{1}{r^{4}}\right\rangle$ value as is given by Born $1960 \mathrm{~K}$, whose values are:

$$
\left\langle\frac{1}{r^{4}}\right\rangle=Z^{4} \times \frac{3-\frac{k^{2}}{n^{2}}}{2 a_{0}^{4} n^{3} k^{5}}
$$

The $k$ parameter is $k \leq n$ and $Z=1$ for neutral atoms. The average quantity $\left\langle\frac{1}{r^{4}}\right\rangle$ goes towards the hydrogen value $\frac{1}{a_{0}^{4} n^{8}}$ when $k \rightarrow n$, for $n=1$ and $k=n$, with $Z=1$ this quantity reaches its maximum value: $\frac{1}{a_{0}^{4} \times n^{8}}, a_{0}$ being the Bohr radius $a_{0}=\frac{\hbar^{2}}{m_{e} e^{2}}$, and the ionization potential $I_{H}=\frac{e^{2}}{a_{0}}$. The quantity $\frac{1}{a_{0}^{4} n^{8}}$ is obtained with $n=1$, and $k=n=1$, these are the quantum numbers of the deepest level of hydrogen in the non-relativistic theory of this atom.

It is easy to define the polarization term for hydrogen atom, that $Z=1$, and the hydrogenic atom is obtained with: $Z \geq 2$ that is:

$$
U_{H}(r, n)=-\frac{\alpha_{D} e^{2}}{2 n^{8} a_{0}} \text { for hydrogen, it is known that } \alpha_{D}=\frac{9}{2} \times a_{0}^{3} \text {. Let's define }
$$
the modified potential seen by an alkaline atom, introducing the corrected interaction potential. That is:

$$
U_{\text {Alk }}\left(r, n_{*}\right)=-\frac{\alpha_{D^{\prime}} e^{2}}{2 n_{*}^{8} a_{0}}, \quad \alpha_{D^{\prime}} \text { given in units of } a_{0}^{3} \text { with } n_{*}=n-\delta \text {, thus }
$$

$n_{*} \leq n$. The polarizabilities of alkaline atoms are always greater than the hydrogen value (see Table 1 ). This implies:

$$
U_{A l k}(r) \geq U_{H}(r) \text {. }
$$

$U_{\text {Alk }}\left(r, n_{*}\right)$ can be very important for low $n$ states, $(n \leq 2)$. 
Table 1. Table of static dipole polarizabilities of neutral elements taken [3].

\begin{tabular}{cccc}
\hline Atom & $\alpha_{D^{\prime}}$ au & errors & L state \\
\hline $\mathrm{H}$ & 4.50 & 0 & ${ }^{1} S$ \\
$\mathrm{Li}$ & 164.1125 & $5 \times 10^{-4}$ & ${ }^{2} S_{1 / 2}$ \\
$\mathrm{Mg}$ & 71.3 & \pm 0.4 & ${ }^{1} S$ \\
$\mathrm{Na}$ & 162.7 & \pm 0.5 & ${ }^{1} S$ \\
$\mathrm{Cs}$ & 400.9 & \pm 0.7 & ${ }^{1} S$ \\
$\mathrm{~K}$ & 289.1 & \pm 0.3 & ${ }^{1} S$ \\
$\mathrm{Ca}$ & 160.8 & \pm 4 & ${ }^{1} S$ \\
\hline
\end{tabular}

Let's define the ratio of these two energies:

$$
\begin{gathered}
R=\frac{U_{A l k}\left(r, n_{*}\right)}{U_{H}(r, n)} \\
R=\frac{n^{8}}{n_{*}^{8}} \times \frac{\alpha_{D^{\prime}}}{\alpha_{D}}
\end{gathered}
$$

Thus the maximal value of $R$ is obtained for: $n=1$.

$$
R_{\max }=\frac{1}{n_{*}^{8}} \times \frac{\alpha_{D^{\prime}}}{\alpha_{D}}
$$

This quantity is a measure of the polarization potential for alkaline atoms, in units of the polarization potential for hydrogen. $R_{\max }$ is a ratio of two energies, it is easy to transform it:

$$
R_{\max }=\frac{T_{1}}{T_{2}}=\frac{2 \alpha_{D^{\prime}}}{9 n_{*}^{8}}
$$

Making the assumption that the alkaline atoms in the molecular clouds are in thermal equilibrium with the accepted values of the molecular clouds, that is $T_{1} \approx T_{\text {clouds }}$ and $50 \mathrm{~K} \geq T_{\text {clouds }} \leq 500 \mathrm{~K}$, it is possible to deduce the values $n_{*}$ corresponding to a peculiar cloud temperature ${ }^{1}$.

\section{Adapting the Alkaline Structure to Cold Interstellar Matter}

The purpose is to show how atomic structure effects (these atoms for which exist quantum defects [5]), could be found in clouds or HI regions.

Let' us consider the Lithium atom, with $\alpha_{D_{L i}}=164.11 \times a_{0}^{3}$ and use $T \approx 100 \mathrm{~K}$ or less, it should be used the following simple formula, using $T_{\text {clouds }}=T_{1}=100 \mathrm{~K}$, equating the polarization potential energy to this thermal energy, $\left|U_{A l k}(r)\right|=\frac{1}{2} \alpha_{D^{\prime}} \frac{158007.11}{n_{*}^{8}}=100$ where $n_{*}$ is given by solving the equation: ${ }^{1}$ au stands for atomic unit and the usual definition for hydrogen $I_{H}=\frac{e^{2}}{a_{0}}=13.616 \mathrm{eV}$ is used or $I_{H}=\frac{1}{2}$ au or in K units $I_{H}=13.616 \times 11604.11=158007 \mathrm{~K}$. 


$$
\begin{gathered}
\frac{1}{2} \alpha_{D_{L i}} \frac{158007.1}{n_{*}^{-8}}=100 \\
n_{*}=\left(\frac{164.11}{200} \times 158007.1\right)^{\frac{1}{8}} \\
n_{*}=4.35
\end{gathered}
$$

These equations are applied to any atoms once their static polarizability: $\alpha_{D^{\prime}}$ is inserted:

$$
\begin{gathered}
\frac{1}{2} \alpha_{D^{\prime}} \frac{158007.1}{n_{*}^{-8}}=T \\
n_{*}(T)=\left(\frac{\alpha_{D^{\prime}}}{2 T} \times 158007.1\right)^{\frac{1}{8}}
\end{gathered}
$$

It is easy to consider the $\mathrm{H}$ atom, for the same temperature $T=100 \mathrm{~K}$, and consider for such value the principal quantum number $n$ obtained by solving:

$$
\begin{gathered}
\frac{9}{4} \frac{158007.1}{n^{-8}}=100 \\
n=\left(\frac{9}{4} \times 1580.071\right)^{\frac{1}{8}} \\
n=2.77
\end{gathered}
$$

The principal quantum number for the $\mathrm{H}$ atom is rounded to $n=3$.

The static polarizability of the atoms that we consider are always: $\alpha_{D^{\prime}} \geq \frac{9}{2}$, thus for a fixed temperature, $T_{\text {clouds }}$ the state $n$ of the hydrogen atom will be such: $n<n_{*}$.

A simple rule can be set from Equation (5), giving a value for $T_{\max }=T_{2}$, that is numerically solving for Lithium element, for a temperature: $T_{1}=T_{\text {clouds }}=100 \mathrm{~K}$ with $R_{\max }=\frac{2 \alpha_{D^{\prime}}}{9 n_{*}^{8}}$

$$
\begin{gathered}
U_{A l k}\left(r, n_{*}\right)=\frac{1}{2} \alpha_{D^{\prime}} \frac{I_{H}}{n_{*}^{8}} \\
R=\frac{U_{A l k}\left(r, n_{*}\right)}{U_{H}(r)} \\
R=\frac{T_{1}}{T_{2}} \\
\frac{T_{1}}{T_{2}}=\frac{100}{T_{2}} \\
\frac{T_{1}}{T_{2}}=\frac{2 \times 164.1}{9 n_{*}^{8}} \\
T_{2}=\frac{T_{1}}{R}
\end{gathered}
$$




$$
\begin{gathered}
T_{2}=\frac{900 \times n_{*}^{8}}{164.1 \times 2} \\
n_{*}=4.35 \\
T_{2}=351575 \mathrm{~K}
\end{gathered}
$$

This temperature $T_{2}$ corresponds to an energy in $\mathrm{eV} \quad E_{2}=2.22 \mathrm{eV}$, considering that $\mathrm{H}$ atoms, are part of molecular clouds of temperature $T_{\text {clouds }}=100 \mathrm{~K}$, they can be found in a state of maximum $n=3$, while the alkaline atom Lithium has a quantum number: $n_{*}=4.35>n$. A Boltzmann factor $X_{B}$ is evaluated to meet the statistical equilibrium for $\mathrm{H}$ and $\mathrm{Li}$ atom.

The energy $E=k_{B} \times T_{\text {clouds }}$ for $T_{\text {Clouds }}=100 \mathrm{~K}$ can be changed in atomic units: that is $1 \mathrm{eV}$ corresponds to $11,604 \mathrm{~K}$, thus $T_{\text {Clouds }}$ corresponds to $0.087 \mathrm{eV}$, although the following equations are sketched in Joule unit give (thus using the Boltzmann constant $k_{B}=1.3806 \times 10^{-23} \mathrm{~J} \cdot \mathrm{T}^{-1}$ ), thus $E=k_{B} \times 100=1.3806 \times 10^{-21} \mathrm{~K}$.

The Bolztmann factor $X_{B}$ can be calculated for the two atoms populations $\mathrm{H}$ atom and any atom whose polarizability is known. It is useful to define the dipole moment $D=e \times a_{0}=8.478353 \times 10^{-30} \mathrm{C} \cdot \mathrm{m}$.

The low temperatures of the clouds make $X_{B}=1$, that is manipulating $X_{B}=e^{-}\left(\frac{U_{L i}-U_{H}}{k b T_{\text {clouds }}}\right)=e\left(\frac{k b T_{\text {clouds }}}{U_{L i}-U_{H}}\right)$, the quantity $k b T_{\text {clouds }}$, is small enough to make $X_{B}=1$.

Except for the situation where $U_{L i}-U_{H}=0$, leading to the following relation: for $\alpha_{D^{\prime}}=164.1$ au:

$$
n_{*}=n \times\left(\frac{4 \alpha_{D^{\prime}}}{9}\right)^{\frac{1}{8}}
$$

For Li element, the and $n=3$ this gives: $n_{*}=1.709 \times n=5.127$.

Even so the low temperature of the clouds, dividing by $k_{B} T$ makes $X_{B}=1$.

$$
\begin{gathered}
\left\langle U_{A l k}\left(r, n_{*}=4.35\right)\right\rangle=U_{A l k}\left(r, n_{*}=4.35\right) \times e^{-\beta U_{L i}} \\
U_{H}(r, n)=\frac{9 D^{2}}{4 n^{8}} \\
\left\langle U_{H}(r, n=3)\right\rangle=U_{H}(r, n=3) \times e^{-\beta U_{H}} \\
\frac{U_{L i}=\frac{\alpha_{L i} D^{2}}{2 \times n_{*}^{8}}}{\left\langle U_{L i}\left(r, n_{*}=4.35\right)\right\rangle}=\frac{U_{L i}\left(r, n_{*}=4.35\right)}{U_{H}(r, n=3)} e^{-}\left(\frac{U_{L i}-U_{H}}{k b T_{\text {clouds }}}\right) \\
X_{B}=e^{-}\left(\frac{U_{L i}-U_{H}}{k b T_{\text {clouds }}}\right) \\
X_{B}=1 \\
\left\langle U_{L i}\left(r, n_{*}\right)\right\rangle \\
\left\langle U_{H}(r, n)\right\rangle
\end{gathered}
$$


It can be generalised for any static polarizability:

$$
\begin{gathered}
U_{\text {Alk }}\left(r, n_{*}\right)=\frac{1}{2} \alpha_{D^{\prime}} \frac{I_{H}}{n_{*}^{8}} \\
R=\frac{U_{\text {Alk }}\left(r, n_{*}\right)}{U_{H}(r)} \\
T_{1}=T_{\text {clouds }} \\
R=\frac{T_{1}}{T_{2}}=\frac{T_{\text {clouds }}}{T_{2}} \\
n_{*}=\left(\frac{\alpha_{D^{\prime}} \times 158007.1}{2 \times T_{\text {clouds }}}\right)^{\frac{1}{8}} \\
T_{2}=\frac{9 \times T_{\text {clouds }} \times n_{*}^{8}}{2 \alpha_{D^{\prime}}} \\
T_{2}=\frac{9 \times 158007.1}{4} \\
T_{\text {max }}=\alpha_{D^{\prime}} \times 79003.5 \\
r_{*}=n_{*}^{2} a_{0} \\
(T)=\left(\frac{T_{\text {max }}}{T}\right)^{\frac{1}{8}}
\end{gathered}
$$

Figure 1 shows how the quantum numbers $n_{*}$ vary with temperatures of the clouds for the Lithium element, it has the greatest polarizability $\alpha_{L i}=164.1$ au.

\section{Transitions to Be Seen}

Interpreting the results in Figure 1 shows that could exist alkaline atoms, in their not so high or low states, there will be a difference with hydrogen in HI regions,

\section{$n_{*}$ quantum number Lithium Polarizability $\alpha \mathrm{D}=164.1$}

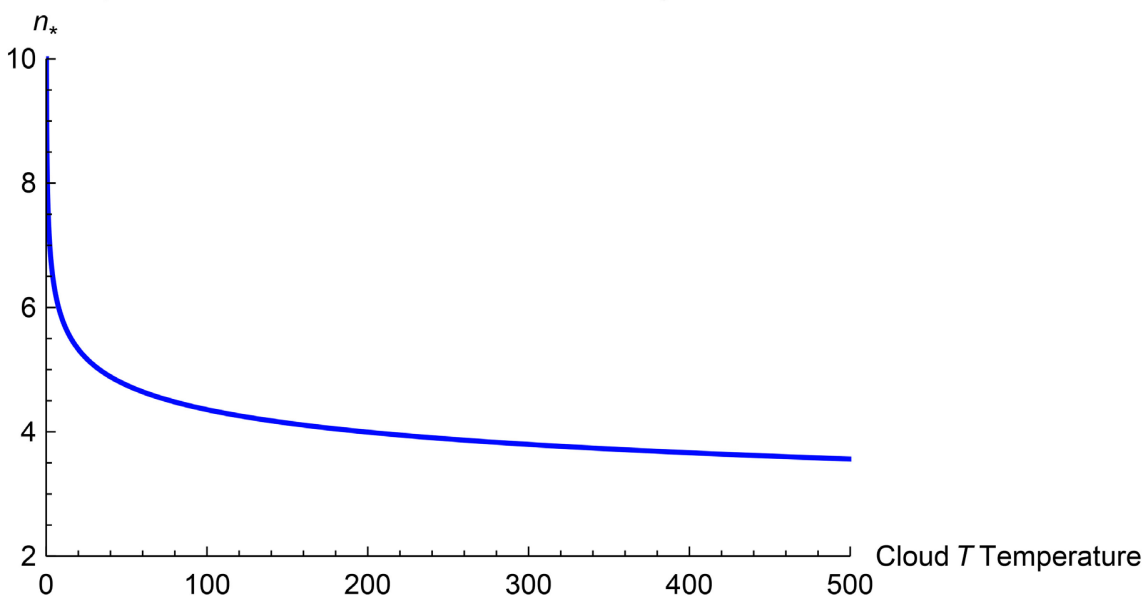

Figure 1. Effective quantum numbers $n_{*}$ as a function of low temperatures $T$ in clouds $20 \mathrm{~K} \leq T \leq 500 \mathrm{~K} . \alpha D$ is given in au that is $a_{0}^{3}$. 
where hydrogen lines exist. In fact, the abundance of elements different from hydrogen is around $\frac{N_{a l k}}{N_{H}} \lesssim 1 \%$.

Depending on the wavelengths seen in molecular clouds emitted by these alkalines could be $n_{*_{i}} \rightarrow n_{* f}=n_{i^{*}}-1$, like $7-\delta_{p} \leq n_{*_{i}} \leq 10-\delta_{p} \rightarrow n_{* f}=2-\delta_{s}$ for a $p \rightarrow s$ transition. If some of these atoms are in thermal equilibrium, it is possible to calculate the fraction of these atoms having the energy:

$$
\begin{gathered}
E_{n, \delta}=-\frac{1}{2} \times \frac{1}{(n-\delta)^{2}} \\
E_{n, \delta}=-\frac{1}{2} \times \frac{1}{n_{*}^{2}}
\end{gathered}
$$

Here the $Z$ charge parameter can be defined for all neutral alkaline elements, ( $Z=1$ ), the ions with $Z>1$ never exist because of the low temperatures of molecular clouds (except if one considers the possible existence of parts of higher temperatures in the molecular clouds).

It is possible to produce with such equilibrium distribution the number of such states. These equations need some comment, when one wants to calculate these partition functions we will use the factor $\beta$ defined in [6] using:

These equations are useful for the conversion of the thermal energy $k_{B} T$ in $\mathrm{eV}$ and in atomic units.

The factor $\beta^{-1}=k_{B} \times T=\frac{T}{11604} \mathrm{eV}$.

Thus a temperature $T_{\text {clouds }}=100 \mathrm{~K}$ corresponds to $\beta^{-1}=0.0086 \mathrm{eV}$ or energy in au $\beta^{-1}=\frac{0.0086}{27.23}=3.16 \times 10^{-4}$ au .

$$
\begin{gathered}
I_{H}=\frac{e^{2}}{a_{0}}=13.616 \mathrm{eV} \\
E_{H}=2 \times I_{H}=27.23 \mathrm{eV} \\
E_{H}=1 \mathrm{a} . \mathrm{u} \\
T_{H}=11604 \times 13.616=158529.2 \mathrm{~K} \\
\beta=\frac{11604}{T}
\end{gathered}
$$

Tables of quantum defects or effective quantum numbers for such atoms or ions can be found in Topbase database: [5]. It is necessary to take into account the level distribution of alkaline atoms in such a way that the partition function is not a divergent sum, (the thing that happens when a negative sign exists in the energy expression):

$$
E_{n, \delta}(T)=-\frac{1}{2} \times \frac{1}{(n-\delta)^{2}}
$$

It is also important to take into account the $\mathrm{n}$ sub levels degeneracy: $g_{n}=2 \times n^{2}$ for each level from $n_{*}$. The assumption implied here is that the po- 
larization potential does not affect the number of sub levels that exists for pure Coulomb states. $g_{n}$ with the spin two states for electron $g_{e}=2$.

Figure 2 shows how the quantum numbers $n_{*}$ vary with the temperatures of the clouds, these depend on the static polarizabilities $\alpha_{D}$ of the atoms.

The correct value of Cons in the atomic unit Cons $=\frac{1}{2}$ au.

Thus Cons $=13.616 \mathrm{eV}$ or in Joule

$$
\text { Cons }=13.616 \times 1.602 \times 10^{-19}=2.181 \times 10^{-18} \mathrm{~J} \text {. }
$$

$$
E_{n *}(T)=g_{n} \frac{e^{\frac{\text { Cons }}{k_{B} T}\left(1-\frac{1}{n-\delta^{2}}\right)}}{Z(T)}
$$

The Maxwell-Boltzmann distribution of such atoms is then:

$$
\begin{gathered}
\frac{\mathrm{d} E_{n, \delta}}{\mathrm{d} n}=\text { Cons } \times \frac{2}{(n-\delta)^{3}} \\
Z(T)=\int_{0}^{\infty} E d_{n_{*}} \mathrm{~d} E_{n_{*}} Z(T)=\int_{0}^{\infty} E d_{n_{*}} \frac{\mathrm{d} E_{n, \delta}}{\mathrm{d} n} \mathrm{~d} n
\end{gathered}
$$

Figure 3 shows how the normalized $N(T)=2 n^{2} \frac{e^{\frac{\text { Cons }}{k_{B} T}\left(1-\frac{1}{n-\delta^{2}}\right)}}{Z(T)}$ varies with principal quantum numbers from $n=1 \rightarrow n=20$ and quantum defects $\delta_{l} \quad 0 \rightarrow 2$.

\section{The Partition Function for Alkaline Atoms: Mg, $\mathrm{Na}, \mathrm{Li}, \mathrm{K}$, Ca, Cs}

Let' us consider each different species of alkaline atoms, $\mathrm{Mg}, \mathrm{Na}, \mathrm{Li}, \mathrm{Cs}, \mathrm{K}, \mathrm{Ca}$. $U_{i}, i=1,2,3,4,5$. For the Caesium element $(\mathrm{NZ}=55)$, quantum defects do not

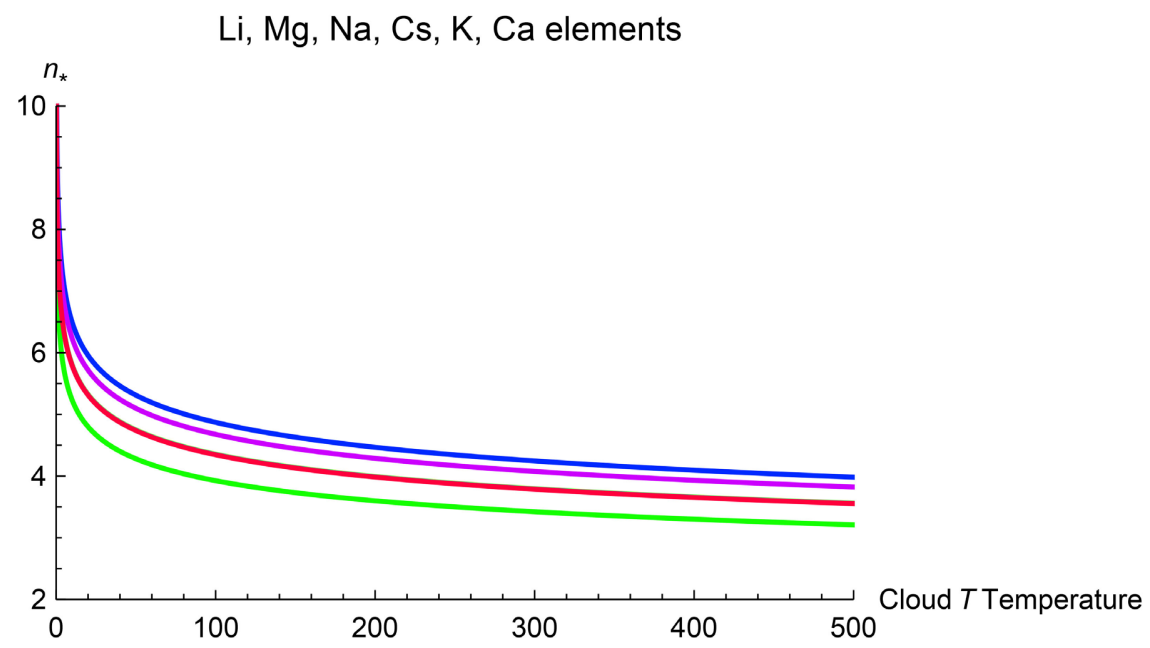

Figure 2. Effective quantum numbers $n_{*}$ as a function of low temperatures $T$ in clouds $20 \mathrm{~K} \leq T \leq 500 \mathrm{~K}$ for $\mathrm{Li}, \mathrm{Mg}, \mathrm{Na}, \mathrm{Cs}, \mathrm{K}$, Ca polarisabilities. The polarizabilities of these atoms are given in Table 1 . Green curve concerns $\mathrm{Mg}$ element, red curve $\mathrm{Ca}$, purple $\mathrm{K}$, blue $\mathrm{Mg}$, other elements curves are indistinguishable. 


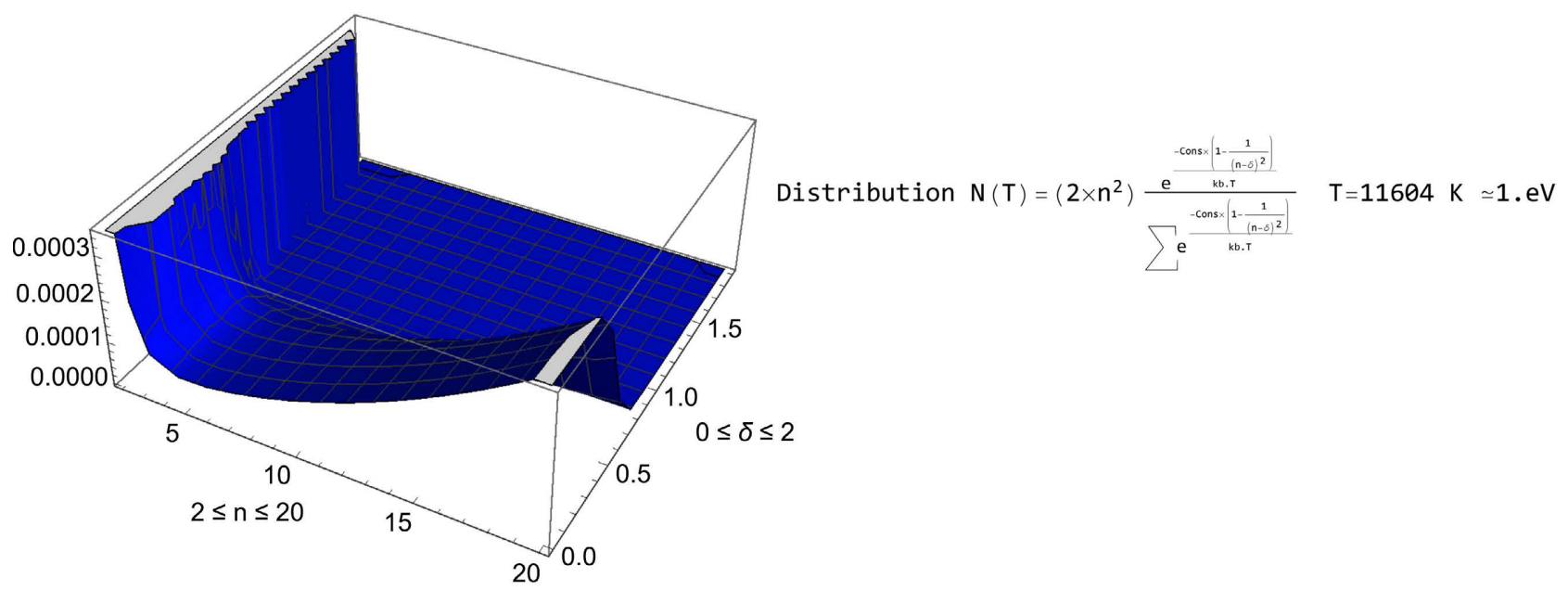

Figure 3. 3D plot giving the normalized distribution $N(T)$ of the atomic population for a temperature $T=11604 \mathrm{~K} \approx 1 \mathrm{eV}$. Effective quantum defects vary as $0 \leq \delta \leq 1.9$ ( $\delta=0$ stands for hydrogen). $\delta=1.9$ is a large quantum defect producing deep energy level thus $N(T)$ is very small. The other axis puts the numbers $n: 1 \leq n \leq 20$. The first edge of this $3 \mathrm{D}$ plot gives the hydrogen population $N_{H}(T)$ for a $1 \mathrm{eV}$ excitation energy.

exist at present, at the author knowledge it is not possible to calculate the Boltzmann $N(T)$ distribution as shown below for elements $\mathrm{Mg}, \mathrm{Na}, \mathrm{Li}, \mathrm{K}, \mathrm{Ca}$, except when:

$\Delta E_{n m}=E_{n}-E_{m}$ are $n S \rightarrow m P$ observed transitions. It can still be evaluated replacing $\Delta E_{n m}=\frac{1}{2}\left(\frac{1}{\left(n-\delta_{s}\right)^{2}}-\frac{1}{\left(m-\delta_{p}\right)^{2}}\right)$ by $\Delta E_{n m}=\frac{h c}{\lambda_{n m}}$ where $\lambda_{n m}$ are given in the NIST database, at the Caesium entry. Here is the partition function for these alkaline atoms: [6]

$$
\begin{gathered}
E i_{n, i}(T)=g_{n} \frac{e^{\frac{\text { Cons }}{k_{B} T}\left(U_{i}-\frac{U_{i}}{n-\delta^{2}}\right)}}{Z(T)} \\
\frac{\mathrm{d} E_{n, \delta}}{\mathrm{d} n}=\operatorname{Cons} \times \frac{2}{(n-\delta)^{3}} \\
Z(T)=\int_{0}^{\infty} E i_{n *} \mathrm{~d} E_{n_{*}} \\
Z(T)=\int_{0}^{\infty} E i_{n_{*}} \frac{\mathrm{d} E_{n, \delta}}{\mathrm{d} n} \mathrm{~d} n
\end{gathered}
$$

\section{Radiating Power $P_{i j}(\omega)$ for the $i \rightarrow j$ Transition in Interstellar Matter}

It is possible to use the cell radiating power for quantum processes such as $a b$ sorption/ emission of light by atoms. This has been done in a very exhaustive and general matter for the Ly- $\alpha$ line [8].

This is interesting to use this formula valid for one excited atom in an identified $i \rightarrow j$ transition: $P(\omega)$. 


$$
\begin{gathered}
P(\omega)=\hbar \cdot P_{i j} \cdot \delta\left(\omega-\omega_{i j}\right) \\
P(\omega)=\frac{4 \omega^{4}}{3 c^{3}} \delta\left(\omega-\omega_{i j}\right)\left|\left\langle\Psi_{i}|\vec{d}| \Psi_{j}\right\rangle\right|^{2}
\end{gathered}
$$

The total radiating power is obtained when the oscillator strengths: $\left|\left\langle\Psi_{i}|\vec{d}| \Psi_{j}\right\rangle\right|^{2}$ of the $i \rightarrow j$ is calculated, such atomic data are now available (with a 10\% agreement) in [5] and [7] these data take into account atomic structure:

NZ number of protons and NE number of electrons for each atomic species $\mathrm{Mg}, \mathrm{Na}, \mathrm{Li}, \mathrm{Ca}$, and fortunately atomic data exists for high states of atoms $\left|\Psi_{i}\right\rangle$ and $\left|\Psi_{j}\right\rangle$ each element has characteristic quantum numbers for a $\Delta L=1$ transition these are $\delta_{s}$ and $\delta_{p}$ quantum defects, $n_{i^{*}}=n_{i}-\delta_{s}$ and $n_{f^{*}}=n_{i}-\delta_{p}$ are the effective quantum numbers ${ }^{2}$ (Tables 2-6).

Table 2. Atomic data used for lithium atomic number $Z=3$.

\begin{tabular}{cccccc}
\hline Element & $n_{* i}=n-\delta_{s}{ }^{\mathrm{a}}$ & $n_{* f}-\delta_{p}{ }^{\mathrm{b}}$ & $\delta_{s}$ & $\delta_{p}$ & gf $^{\mathrm{c}}$ \\
\hline $\mathrm{Li}$ & 4.60 & 4.955 & 0.398 & 0.044 & 4.10 \\
$\mathrm{Li}$ & 5.60 & 5.955 & 0.398 & 0.046 & 4.91 \\
$\mathrm{Li}$ & 6.60 & 6.995 & 0.397 & 0.047 & 5.72 \\
$\mathrm{Li}$ & 7.60 & 7.955 & 0.397 & 0.048 & 6.52 \\
$\mathrm{Li}$ & 8.60 & 8.995 & 0.397 & 0.049 & 6.52
\end{tabular}

${ }^{a} P_{T}(\omega)$ has indeed the dimension of a power: $\frac{\mathrm{d} E}{\mathrm{~d} t}$ when one uses the good units for the line strength that is $D^{2} \propto\left(e a_{0}\right)^{2}$, in fact, it can be written: $D^{2}=\frac{e^{2}}{a_{0}} a_{0}^{3}$, it is then straightforward to check that $P_{T}(\omega)$ has a dimension of energy by a unit of time; ${ }^{\mathrm{b}} n_{*_{i}}$ is the effective quantum number for down-level; ${ }^{\mathrm{c}}$ The data for gf oscillator strength are taken from [7].

Table 3. Atomic data used for sodium atomic number $Z=11$.

\begin{tabular}{cccccc}
\hline Element & $n_{* i}=n-\delta_{s}{ }^{\mathrm{a}}$ & $n_{* f}-\delta_{p}{ }^{\mathrm{b}}$ & $\delta_{s}$ & $\delta_{p}$ & gf $^{\mathrm{c}}$ \\
\hline $\mathrm{Na}$ & 4.66 & 5.154 & 1.337 & 0.845 & 4.63 \\
$\mathrm{Na}$ & 5.66 & 6.155 & 1.336 & 0.844 & 5.47 \\
$\mathrm{Na}$ & 6.66 & 7.154 & 1.336 & 0.843 & 6.30 \\
$\mathrm{Na}$ & 7.66 & 8.664 & 1.336 & 0.843 & 7.13 \\
$\mathrm{Na}$ & 8.66 & 8.156 & 1.335 & 0.842 & 0.30
\end{tabular}

${ }^{\text {a }} P_{T}(\omega)$ has indeed the dimension of a power: $\frac{\mathrm{d} E}{\mathrm{~d} t}$ when one uses the good units for the line strength that is $D^{2} \propto\left(e a_{0}\right)^{2}$, in fact, it can be written: $D^{2}=\frac{e^{2}}{a_{0}} a_{0}^{3}$, it is then straightforward to check that $P_{T}(\omega)$ has a dimension of energy by a unit of time; ${ }^{\mathrm{b}} n_{* i}$ is the effec-

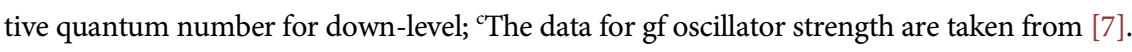

${ }^{2}$ The K Potassium element NZ $=\mathrm{NE}=19$ is not referenced in [7], Caesium Cs oscillator strengths are not available from because of the atomic number $\mathrm{NE}=55$, in fact the Topbase data stops for NE $\geq 27$, the last element given is $\mathrm{NE}=26$ that is neutral iron Fe. 
Table 4. Atomic data used for magnesium atomic number $Z=12$.

\begin{tabular}{cccccc}
\hline Element & $n_{* i}=n-\delta_{s}{ }^{\mathrm{a}}$ & $n_{* f}-\delta_{p}{ }^{\mathrm{b}}$ & $\delta_{s}$ & $\delta_{p}$ & gf $^{\mathrm{c}}$ \\
\hline $\mathrm{Mg}$ & 3.49 & 4.73 & 1.507 & 1.268 & 1.69 \\
$\mathrm{Mg}$ & 4.47 & 5.74 & 1.526 & 1.258 & 2.22 \\
$\mathrm{Mg}$ & 5.47 & 6.74 & 1.521 & 1.253 & 2.10 \\
$\mathrm{Mg}$ & 6.48 & 7.75 & 1.519 & 1.249 & 3.49 \\
$\mathrm{Mg}$ & 7.48 & 8.75 & 1.517 & 1.247 & 0.12 \\
\hline
\end{tabular}

${ }^{a} P_{T}(\omega)$ has indeed the dimension of a power: $\frac{\mathrm{d} E}{\mathrm{~d} t}$ when one uses the good units for the line strength that is $D^{2} \propto\left(e a_{0}\right)^{2}$, in fact, it can be written: $D^{2}=\frac{e^{2}}{a_{0}} a_{0}^{3}$, it is then straightforward to check that $P_{T}(\omega)$ has a dimension of energy by a unit of time; ${ }^{\mathrm{b}} n_{*_{i}}$ is the effective quantum number for down-level; ${ }^{\mathrm{c}}$ The data for gf oscillator strength are taken from [7].

Table 5. Atomic data used for potassium $\mathrm{K}$ atomic number $Z=19$.

\begin{tabular}{cccccc}
\hline Element & $n_{* i}=n-\delta_{s}{ }^{\mathrm{a}}$ & $n_{* f}-\delta_{p}{ }^{\mathrm{b}}$ & $\delta_{s}$ & $\delta_{p}$ & gf $^{\mathrm{c}}$ \\
\hline $\mathrm{K}$ & 3.675 & 3.960 & 2.324 & 1.040 & 1.26 \\
$\mathrm{~K}$ & 4.705 & 4.874 & 2.294 & 1.126 & 1.01 \\
$\mathrm{~K}$ & 5.703 & 5.755 & 2.296 & 1.244 & 0.37 \\
$\mathrm{~K}$ & 6.703 & 6.619 & 2.296 & 1.381 & 0.68 \\
$\mathrm{~K}$ & 8.703 & 8.417 & 2.296 & 1.583 & 2.64 \\
\hline
\end{tabular}

${ }^{\text {a }} P_{T}(\omega)$ has indeed the dimension of a power: $\frac{\mathrm{d} E}{\mathrm{~d} t}$ when one uses the good units for the line strength that is $D^{2} \propto\left(e a_{0}\right)^{2}$, in fact, it can be written: $D^{2}=\frac{e^{2}}{a_{0}} a_{0}^{3}$, it is then straightforward to check that $P_{T}(\omega)$ has a dimension of energy by a unit of time; ${ }^{\mathrm{b}} n_{*_{i}}$ is the effective quantum number for down-level; ${ }^{c}$ The data for gf oscillator strength are taken from [7].

Table 6. Atomic data used for calcium Ca atomic number $Z=20$.

\begin{tabular}{cccccc}
\hline Element & $n_{*_{i}}=n-\delta_{s}{ }^{\mathrm{a}}$ & $n_{* f}-\delta_{p}{ }^{\mathrm{b}}$ & $\delta_{s}$ & $\delta_{p}$ & $\mathrm{gf}^{\mathrm{c}}$ \\
\hline $\mathrm{Ca}$ & 3.675 & 3.960 & 2.324 & 1.040 & 1.26 \\
$\mathrm{Ca}$ & 4.705 & 4.874 & 2.294 & 1.126 & 1.01 \\
$\mathrm{Ca}$ & 5.703 & 5.755 & 2.296 & 1.244 & 0.37 \\
$\mathrm{Ca}$ & 6.703 & 6.619 & 2.296 & 1.381 & 0.68 \\
$\mathrm{Ca}$ & 8.703 & 8.417 & 2.296 & 1.583 & 2.64 \\
\hline
\end{tabular}

${ }^{a} P_{T}(\omega)$ has indeed the dimension of a power: $\frac{\mathrm{d} E}{\mathrm{~d} t}$ when one uses the good units for the line strength that is $D^{2} \propto\left(e a_{0}\right)^{2}$, in fact, it can be written: $D^{2}=\frac{e^{2}}{a_{0}} a_{0}^{3}$, it is then straightforward to check that $P_{T}(\omega)$ has a dimension of energy by a unit of time; ${ }^{\mathrm{b}} n_{* i}$ is the effective quantum number for down-level; ${ }^{c}$ The data for gf oscillator strength are taken from [7]. 


\section{Links to Oscillator Strengths of $\mathrm{Mg}, \mathrm{Na}, \mathrm{Li}, \mathrm{Cs}, \mathrm{K}, \mathrm{Ca}$}

The oscillator strength $f_{i j}$ and of the line strengths: $S_{i j}$ formulae are given using: $\left\langle\Psi_{i}\right|=\left\langle 1 m_{1}\right|$ and $\left|\Psi_{j}\right\rangle=\left|2 m_{2}\right\rangle$.

$$
\begin{gathered}
f_{12}=\frac{2}{3} \frac{m_{e}}{\hbar^{2}}\left(E_{2}-E_{1}\right) \sum_{\alpha=x, y, z}\left|\left\langle 1 m_{1}\left|R_{\alpha}\right| 2 m_{2}\right\rangle\right|^{2} \\
f_{12}=\frac{2}{3}(\Delta E)\left|\left\langle 1 m_{1}|\tilde{D}| 2 m_{2}\right\rangle\right|^{2}(\mathrm{au}) \\
S_{12}=\sum_{\alpha=x, y, z}\left|\left\langle 1 m_{1}\left|e \cdot R_{\alpha}\right| 2 m_{2}\right\rangle\right|^{2} \\
S_{12}=\left|\left\langle 1 m_{1}|\tilde{D}| 2 m_{2}\right\rangle\right|^{2} \\
f_{12}=\frac{2}{3}\left(\frac{\Delta E}{g_{1}}\right) S_{12}(\mathrm{au})
\end{gathered}
$$

with the definition of the dipole $\tilde{D}=e \cdot \tilde{R}$, these relations enable these expressions written in au (atomic unit):

$$
\begin{gathered}
P\left(\omega_{i j}\right)=\frac{4 \omega_{i j}^{4}}{3 c^{3}} S_{i j} \\
P\left(\omega_{i j}\right)=\frac{2 \omega_{i j}^{3}}{c^{3}} g_{i} f_{i j}
\end{gathered}
$$

Taking into account the effect of the number of atoms through the line of sight $N_{a t}(h)$ and the Boltzmann distribution of these atomic emitters: (with $Z=1$ ).

$$
\begin{gathered}
N(T)=\left(2 n^{2}\right) \cdot \frac{e^{-\frac{k o n s \cdot Z^{2} \times\left(1-\frac{1}{(n-\delta)^{2}}\right)}{k b T}}}{\sum e^{-\frac{-C o n s \cdot Z^{2} \times\left(1-\frac{1}{(n-\delta)^{2}}\right)}{k b T}}} \\
P_{T}(\omega) \mathrm{d} \Omega=N(T) \times N_{a t}(h) \frac{4 \omega_{i j}^{4}}{3 c^{3}} S_{i j} \frac{\mathrm{d} \Omega}{4 \pi}
\end{gathered}
$$

\section{Special Treatment for Element K Potassium and Cs Caesium}

It is possible to use data of NIST database for lines ${ }^{3}$, and to use the output for oscillator strengths: $g f_{i k}$ and $S_{i k}$ line strengths for known transitions as for instance (Table 7):

It is possible to evaluate quantum defects $\delta_{s}$, and $\delta_{p}$, for the $\mathrm{K}$ element. This is impossible for the Cs element because it has so many electrons although there exist observed and identified transitions provided with the oscillator strengths $g f_{i k}$ [5], this enables anyhow to give an estimate of the emitted power by Cs atoms if of course, these atoms exist in the molecular cloud.

The way to get there is quite simple and easily performed using Mathematica software, the NIST database gives transitions observed and calculated. One ${ }^{3}$ Transitions and gf atomic data exist for K element and Cs element in NIST database [5]. 
Table 7. Cosmic abundance of atoms $\mathrm{Mg}, \mathrm{Ca}, \mathrm{Li}, \mathrm{Na}$.

\begin{tabular}{cc}
\hline Element & Cosmic abundance per $10^{6} \mathrm{H}$ atoms \\
\hline $\mathrm{Mg}$ & $4.0 \times 10^{5}$ \\
$\mathrm{Ca}$ & $6.0 \times 10^{4}$ \\
$\mathrm{Li}$ & 3 \\
$\mathrm{Na}$ & $6.0 \times 10^{4}$ \\
\hline
\end{tabular}

needs a least two transitions, with the same kinetic moment change $L \pm 1$, that is $n s \rightarrow n p$, these transitions are defined in Table 8. Two identified transitions for $\mathrm{K}$ elements suffice to evaluate the $\delta_{s}, \delta_{p}$ quantum defects, more than two will reinforce the following equation solutions.

$$
\begin{aligned}
& \text { Solve }\left(\frac{1}{\left(4-\delta_{p}\right)^{2}}-\frac{1}{\left(5-\delta_{s}\right)^{2}}\right)=\frac{12432.2}{911.76} \\
& \text { Solve }\left(\frac{1}{\left(4-\delta_{p}\right)^{2}}-\frac{1}{\left(6-\delta_{s}\right)^{2}}\right)=\frac{6911}{911.76}
\end{aligned}
$$

Here the ionization potential is given in $\AA$, that is $I_{H}=911.76 \AA$. Solving this set of equations, for the two quantum defects $\left(\delta_{s}, \delta_{p}\right)$, gives several solutions (in fact 8 different sets), 4 give complex solutions to be discarded, and 4 reals and one of these gives reasonable values, that is: $\delta_{s}=2.2069$ and $\delta_{p}=1.7718$.

\section{Links to the Surrounding Medium}

Once atomic parameters are obtained, the following statistic of the emitters is possible, it has two parts:

The first $N_{a t}(h)$ depends on the repartition of the alkaline emitters on the line of sight inside these molecular clouds. The second part is simply the thermal Boltzmann distribution $\mathrm{N}(\mathrm{T})$ of these states, within the clouds. For the function $N_{a t}(h)$ that gives the number of atoms of different species, I shall use the simplest form: neglecting the effects of absorption or emission inside the cloud.

$$
N_{a t}(h)=N_{a} \times h^{3}
$$

Here we shall use data for the number of hydrogen existing in a molecular cloud, the fact that the cosmic abundances of the elements $\mathrm{Ca}$ and $\mathrm{Na}$ are nearly the same is commonly accepted.

$$
N_{H}=2 \times N_{H_{2}}+N_{H I}+N_{H I I}
$$

That means that we account for all the different forms of this element. This gives a number of hydrogen: $N_{H} \approx 3 \times 10^{10}, \mathrm{~m}^{-3}$ while for Li element one uses $N_{L i} \approx 10^{-10} N_{H}$. In this approach of guessing the power emitted by these species: $\mathrm{Mg}, \mathrm{Na}, \mathrm{Li}, \mathrm{K}, \mathrm{Ca}$, we shall not consider opacity of the lines in the molecular cloud, that is absorption/(re)emission processes, this will simplify the calculation of the final results that is the $P_{T}^{a t}(\omega)$. For what concerns the Boltzmann distribution 
Table 8. Atomic data used for Li Na Mg elements.

\begin{tabular}{ccccccc}
\hline Element & $\mathrm{n} \mathrm{s}$ & $\mathrm{n} \mathrm{p}$ & $J \rightarrow J^{\prime}$ & Wavelength $\AA$ & $g f_{12}$ & $S_{12}(\mathrm{au})$ \\
\hline $\mathrm{Li}$ & $4 \mathrm{~s}$ & $5 \mathrm{p}$ & $\frac{1}{2} \rightarrow \frac{1}{2}$ & $24,978$. & $3.17 \times 10^{-4}$ & 0.05 \\
$\mathrm{Na}$ & $6 \mathrm{~s}$ & $5 \mathrm{p}$ & $\frac{1}{2} \rightarrow \frac{1}{2}$ & $16,373$. & $1.60 \times 10^{-1}$ & 2.54 \\
$\mathrm{Mg}$ & $5 \mathrm{~s}$ & $6 \mathrm{p}$ & $\frac{1}{2} \rightarrow \frac{1}{2}$ & 121,464 & $4.80 \times 10^{-2}$ & 3.39 \\
\hline
\end{tabular}

$N(T)$, the behaviour depends on the different $U_{i}$ ionization potentials see Table 2 and on the defects $\delta_{s}, \delta_{p}$ for the $\mathrm{Mg}, \mathrm{Na}, \mathrm{Li}, \mathrm{Cs}, \mathrm{K}, \mathrm{Ca}$ elements. The distribution $\mathrm{N}(\mathrm{T})$ is illustrated in Figure 4.

\subsection{Emitted Power Estimate from GMC Molecular Cloud for Mg, $\mathrm{Na}$, K, Li Elements}

It is accepted that millimetre wave or submillimetre wave in emission from a molecular cloud, meet an optically thin medium.

It is possible to construct, in a purely theoretical way many lines with high quantum numbers $\left(n_{*} \geq 5\right)$, when the quantum defects are known.

The power emitted at the edge of the molecular clouds, depends on the nature of the atom $(\mathrm{Li}, \mathrm{Na}, \mathrm{K}, \mathrm{Mg})$, through the $S_{i j}$ line strength and of the related $i \rightarrow j$ transition $\omega_{i j}=2 \pi v_{i j}=2 \pi \frac{c}{\lambda_{i j}}$ (Table $9 \&$ Table 10).

It is thus easy to calculate the emitted power for each atomic species:

$$
P_{n m}(\omega) \mathrm{d} \Omega=N(T) \times N_{a t}(h) \frac{64 \pi^{4} c}{3 \lambda_{n m}^{4}} S_{i j} \times\left(e^{2} a_{0}^{2}\right) \frac{\mathrm{d} \Omega}{4 \pi}
$$

\subsection{Telescope Detection of GMC Emitters}

It is admitted that the molecular clouds in infrared or near-infrared wavelengths are optically thin where these high quantum states are optically are to be found (the emitted photons of these alkaline atoms).

The receiving device, being at a distance $D_{\max }=640$ light-years, that is $D_{\max }=6.054 \times 10^{18} \mathrm{~m}$, it is necessary to define the solid angle of the molecular cloud for the observer device that is: $\mathrm{d} \Omega=2 \pi \sin (\theta) \mathrm{d} \theta$, the angle $\theta$ is defined as follows:

$$
\tan \left(\frac{\theta}{2}\right)=\frac{L}{2 D_{\max }}
$$

$L$ is the diameter of the cloud, $L \approx 45$ parsec or $L \approx 147$ ly, numerically $\tan \left(\frac{\theta}{2}\right) \approx 0.114$ rd, considering $\tan (\theta) \approx \theta$ it follows:

$$
\frac{\mathrm{d} \Omega}{4 \pi}=\frac{1}{2} \int_{0}^{\theta} \sin (\theta) \mathrm{d} \theta
$$




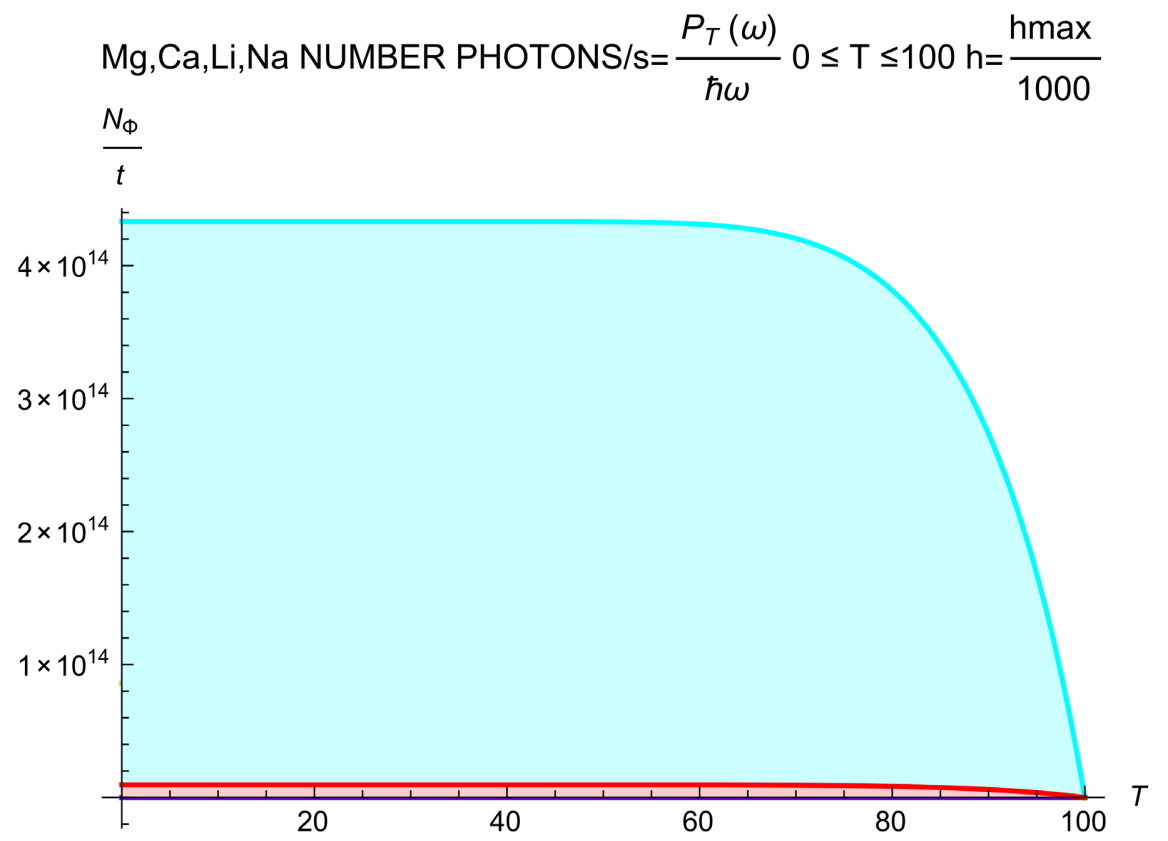

Figure 4. Different colors each alkaline red $\mathrm{Mg} \mathrm{Ca}$ Li give the number of photons by the unit of time $\frac{N_{\phi}}{t}$ this depends on the temperature $T$ of the cloud. These figures give the maximal number of photons to be seen because the quantity $N_{A t} \propto(\text { Volume })^{\frac{1}{3}}=\frac{h_{\max }}{1000}$. $h_{\max }$ is the maximal spatial extension of the cloud.

Table 9. Atomic data used for caesium Cs atomic number $Z=55$ and $\mathrm{K}$ potassium $Z=$ 19.

\begin{tabular}{ccccccc}
\hline Element & $\mathrm{n} \mathrm{s}$ & $\mathrm{n} \mathrm{p}$ & $J \rightarrow J^{\prime}$ & Wavelength $\AA$ & $g f_{12}$ & $S_{12}(\mathrm{au})$ \\
\hline $\mathrm{Cs}$ & $6 \mathrm{~s}$ & $6 \mathrm{p}$ & $\frac{1}{2} \rightarrow \frac{1}{2}$ & 8521.6 & 4.00 & \\
$\mathrm{~K}$ & $5 \mathrm{~s}$ & $4 \mathrm{p}$ & $\frac{1}{2} \rightarrow \frac{1}{2}$ & 12,432 & 0.18 & \\
$\mathrm{~K}$ & $6 \mathrm{~s}$ & $4 \mathrm{p}$ & $\frac{1}{2} \rightarrow \frac{1}{2}$ & 6911.0 & $1.4 \times 10^{-2}$ & \\
$\mathrm{~K}$ & $5 \mathrm{~s}$ & $7 \mathrm{p}$ & $\frac{1}{2} \rightarrow \frac{1}{2}$ & 9954.1 & $2.0 \times 10^{-3}$ & \\
\hline
\end{tabular}

Table 10. Atomic data used for Li Na Mg elements.

\begin{tabular}{ccccccc}
\hline Element & $\mathrm{n} \mathrm{s}$ & $\mathrm{n} \mathrm{p}$ & $J \rightarrow J^{\prime}$ & Wavelength $\AA$ & $g f_{12}$ & $S_{12}(\mathrm{au})$ \\
\hline $\mathrm{Li}$ & $4 \mathrm{~s}$ & $5 \mathrm{p}$ & $\frac{1}{2} \rightarrow \frac{1}{2}$ & $24,978.1$ & $3.1 \times 10^{-4}$ & 0.052 \\
$\mathrm{Na}$ & $6 \mathrm{~s}$ & $5 \mathrm{p}$ & $\frac{1}{2} \rightarrow \frac{1}{2}$ & $16,373.8$ & $1.6 \times 10^{-1}$ & 2.54 \\
$\mathrm{Mg}$ & $5 \mathrm{~s}$ & $6 \mathrm{p}$ & $\frac{1}{2} \rightarrow \frac{1}{2}$ & 121,464 & $4.8 \times 10^{-2}$ & 3.39 \\
\hline
\end{tabular}


Table 11. Predicted observational physical data: received power $P_{T}(\omega)$ and flow of photons $\Phi=\frac{N_{\phi}}{t}$ for $\mathrm{Mg}, \mathrm{Ca}, \mathrm{Li}, \mathrm{Na}$ atomic transitions as a function of the giant molecular cloud Temperature $T$.

\begin{tabular}{|c|c|c|c|c|c|}
\hline Element & $\begin{array}{c}\text { Temperature } \\
T(\mathrm{~K})\end{array}$ & $\begin{array}{c}\text { Wavelength } \\
\text { (A) }\end{array}$ & Power $P_{T}(\omega)$ & $\begin{array}{c}\text { Flow } \\
\text { of photons }\end{array}$ & $\Phi=\frac{N_{\phi}}{t}$ \\
\hline $\mathrm{Mg}$ & 20 & 12.14 . & $3.19 \times 10^{-4}$ & $3.86 \times 10^{14}$ & $3.86 \times 10^{6}$ \\
\hline $\mathrm{Mg}$ & 40 & 12.14 & $3.19 \times 10^{-4}$ & $3.86 \times 10^{14}$ & $3.86 \times 10^{6}$ \\
\hline $\mathrm{Mg}$ & 60 & 12.14 & $3.19 \times 10^{-4}$ & $3.86 \times 10^{14}$ & $3.86 \times 10^{6}$ \\
\hline $\mathrm{Mg}$ & 80 & 12.14 & $3.19 \times 10^{-4}$ & $3.86 \times 10^{15}$ & $3.86 \times 10^{6}$ \\
\hline $\mathrm{Mg}$ & 100 & 12.14 & $3.19 \times 10^{-4}$ & $3.86 \times 10^{15}$ & $3.86 \times 10^{6}$ \\
\hline $\mathrm{Ca}$ & 20 & 5.581 & $7.33 \times 10^{-3}$ & $1.94 \times 10^{16}$ & $1.94 \times 10^{7}$ \\
\hline $\mathrm{Ca}$ & 40 & 5.581 & $7.33 \times 10^{-3}$ & $1.94 \times 10^{16}$ & $1.94 \times 10^{7}$ \\
\hline $\mathrm{Ca}$ & 60 & 5.581 & $7.33 \times 10^{-3}$ & $1.94 \times 10^{16}$ & $1.94 \times 10^{7}$ \\
\hline $\mathrm{Ca}$ & 80 & 5.581 & $7.33 \times 10^{-3}$ & $1.94 \times 10^{16}$ & $1.94 \times 10^{7}$ \\
\hline $\mathrm{Ca}$ & 100 & 5.581 & $7.33 \times 10^{-3}$ & $1.84 \times 10^{16}$ & $1.94 \times 10^{7}$ \\
\hline $\mathrm{Na}$ & 20 & 16.37 & $5.11 \times 10^{-5}$ & $4.30 \times 10^{14}$ & $4.30 \times 10^{5}$ \\
\hline $\mathrm{Na}$ & 40 & 16.37 & $5.11 \times 10^{-5}$ & $4.30 \times 10^{14}$ & $4.30 \times 10^{5}$ \\
\hline $\mathrm{Na}$ & 60 & 16.37 & $5.11 \times 10^{-5}$ & $4.30 \times 10^{14}$ & $4.30 \times 10^{5}$ \\
\hline $\mathrm{Na}$ & 80 & 16.37 & $5.11 \times 10^{-5}$ & $4.30 \times 10^{14}$ & $4.30 \times 10^{5}$ \\
\hline $\mathrm{Na}$ & 100 & 16.37 & $5.11 \times 10^{-5}$ & $4.30 \times 10^{14}$ & $4.29 \times 10^{5}$ \\
\hline $\mathrm{Li}$ & 20 & 524.9 & $9.96 \times 10^{-12}$ & $1.27 \times 10^{8}$ & 0.1271 \\
\hline $\mathrm{Li}$ & 40 & 524.9 & $9.96 \times 10^{-12}$ & $1.27 \times 10^{8}$ & 0.1271 \\
\hline $\mathrm{Li}$ & 60 & 524.9 & $9.96 \times 10^{-12}$ & $1.27 \times 10^{8}$ & 0.1271 \\
\hline $\mathrm{Li}$ & 80 & 524.9 & $9.96 \times 10^{-12}$ & $1.27 \times 10^{8}$ & 0.1271 \\
\hline $\mathrm{Li}$ & 100 & 524.9 & $9.95 \times 10^{-12}$ & $1.27 \times 10^{8}$ & 0.1270 \\
\hline
\end{tabular}

$$
\frac{\mathrm{d} \Omega}{4 \pi}=\frac{1}{2}\left(1-\cos \theta^{2}\right) \approx 0.25\left(\frac{L}{D_{\max }}\right)^{2} \approx 0.0025
$$

${ }^{4}$ The received light emitted from the molecular cloud should be $P_{R}^{i}$, each index $i$, $i=1,2,3,4$ for subsequent elements $\mathrm{Mg}, \mathrm{Ca}, \mathrm{Li}, \mathrm{Na} . \mathrm{R}$ is the radius of the telescope (Table 11 \& Table 12).

$$
\begin{aligned}
& h_{1}=\frac{h_{\max }}{3} \text {, and then for } h_{2}=\frac{h_{1}}{1000} \text {, the fluxes then are in the ratio: } \frac{\Phi_{2}}{\Phi_{1}} \approx 10^{-9} . \\
& P_{R}^{i}=\frac{P_{T}}{4 \pi D_{\text {max }}^{2}} \times \pi R^{2} \times \mathrm{d} \Omega
\end{aligned}
$$

It is assumed that for the following wavelengths in $\mu \mathrm{m}$, the emitting medium is optically thin, it is remarkable that the photons flux is proportional to $V \approx h^{3}$, where at the maximum, $h=h_{\max }$, the following data give an estimate for:

${ }^{4}$ The model GMC given in the table yields a field in the squared degree of 0.0019 nearly 500 times less than a squared degree. 
Table 12. Physical data for molecular clouds used for an estimate of the power to be detected by a spectrograph.

\begin{tabular}{ccc}
\hline Physical GMC data & Quantity & Units \\
\hline Number & 4000 & dimensionless \\
Mass & $2 \times 10^{5}$ & $M_{\odot}$ solar masses \\
Mean diameter & 45 & parsec \\
Projected surface area & 45 & parsec $^{2}$ \\
Volume & $10^{5}$ & parsec $^{3}$ \\
Volume density $\left(H_{2}\right)$ & $3 \times 10^{11}$ & $\mathrm{~m}^{-3}$ \\
Alkaline density $\left(\frac{N_{a l k}}{H_{2}}\right)$ & $5 \times 10^{-3}$ & \\
Mean surface density & $1.5 \times 10^{124}$ & $\mathrm{~m}^{-2}$ \\
Mean separation & 500 & parsec \\
Orion GMC distance $D_{\max }$ to Observer & 640 & light-years
\end{tabular}

$h_{1}=\frac{h_{\max }}{3}$, and then for $h_{2}=\frac{h_{1}}{1000}$, the fluxes then are in the ratio: $\frac{\Phi_{2}}{\Phi_{1}} \approx 10^{-9}$.

\subsection{Physical Data of Giant Molecular Clouds}

Here are some data to be used for receiving the alkaline $n S \rightarrow m P$ transitions to an observer on Earth (or even to the future infrared space telescope JWST) of such emissions from giant molecular clouds.

Figure 4 provides an estimate of the power radiated by atoms of different species for a cloud temperatures range $5 \leq T_{\text {clouds }} \leq 100 \mathrm{~K}$.

\section{Conclusions}

It is shown how alkaline atoms transitions whose structures are described by these set of quantum numbers: $n_{*}=n-\delta$ with quantum defects $\delta$ such as $0 \leq \delta \leq 1.9$ and principal quantum numbers $5 \leq n \leq 9$ can exist in cold molecular clouds where it is found neutral $\mathrm{H}$ atoms and many molecular compounds.

Under the reasonable assumptions of an optically thin media, at the rather high wavelength, and with an abundance of the considered atoms in accordance with cosmological data, it is given for experimental device (such as a large telescope), the number of photons, by second, for each different line whose line strengths are known.

It is obvious to see in the last figure that the photon flux $\Phi=\frac{N_{\Phi}}{t}$ is directly proportional to the length of the spatial extent of the clouds.

To the author's knowledge, these infrared lines are not yet detected but are seen in a future survey of molecular clouds, these should be coming from LEA, little excited atoms. It is probably possible that these radiating neutral atoms could be part of vortices, as the $\mathrm{CO}$ molecules, and then the information to track 
such motions in the clouds is linked to the line profile of these atoms.

\section{Acknowledgements}

The author expresses his thanks to Benoit Albert, Computer Engineer of the LERMA laboratory for his assistance on computer management.

\section{Conflicts of Interest}

The author declares no conflicts of interest regarding the publication of this paper.

\section{References}

[1] Born, M. (1960) The Mechanics of the Atom. pp 163 Ungar Edition.

[2] van Dishoeck, E.F. (2004) ISO Spectroscopy of Gas and Dust: From Molecular Clouds to Protoplanetary Disks. Annual Review of Astronomy and Astrophysics, 42, 119-167. https://doi.org/10.1146/annurev.astro.42.053102.134010

[3] Schwerdtfeger, P. and Nagle, J.K. (2019) 2018 Table of Static Dipole Polarizabilities of the Neutral Elements in the Periodic Table. Molecular Physics, 117, 1200-1225. https://doi.org/10.1080/00268976.2018.1535143

[4] Mitroy, J., Safronova, M.S. and Clark, C.W. (2010) Theory and Applications of Atomic and Ionic Polarisabilities. Journal of Physics B: Atomic, Molecular and Optical Physics, 43, Article No. 202001. http://stacks.iop.org/0953-4075/43/i=20/a=202001 https://doi.org/10.1088/0953-4075/43/20/202001

[5] Kelly, R.L. (2012) NIST Database on Line Consultation. https://www.nist.gov/pml/atomic-spectra-database

[6] Blinder, S.M. (1995) High- and Low-Energy Estimates for the Dirac Equation. Journal of Mathematical Physics, 36, Article No. 991. https://doi.org/10.1063/1.531138

[7] Mendoza, C. (1993) The Iron Project-The Opacity Project IPOPv2. IVIC. PO Box 21827, Caracas 1020A, Venezuela. http://cdsweb.u-strasbg.fr/OP.html

[8] Feautrier, N., Tran Minh, N. and Van Regemorter, H. (1976) The Quantum Unified Theory Applied to the Lyman- $\alpha$ Profile Calculation. Journal of Physics B: Atomic and Molecular Physics (1968-1987), 9, No. 11.

https://doi.org/10.1088/0022-3700/9/11/013 